\title{
28 Research Soure

\section{A Novel Density Peaks Clustering Algorithm Based on K Nearest Neighbors With Adaptive Merging Strategy}

\section{Xiaoning Yuan}

Nuclear Power Institute of China

Hang Yu

Nuclear Power Institute of China

Jun Liang

Zhejiang University

Bing Xu ( $\sim$ bingxu@zju.edu.cn )

State Key Lab of Industrial Control Technology, College of Control Science and Engineering, Zhejiang University, Hangzhou, 310027, China https://orcid.org/0000-0003-3795-7263

\section{Research}

Keywords: density peaks, clustering algorithm, K nearest neighbors, adaptive merging

Posted Date: October 23rd, 2020

DOl: https://doi.org/10.21203/rs.3.rs-95747/v1

License: (c) (i) This work is licensed under a Creative Commons Attribution 4.0 International License. Read Full License

Version of Record: A version of this preprint was published at International Journal of Machine Learning and Cybernetics on August 3rd, 2021. See the published version at https://doi.org/10.1007/s13042-02101369-7. 


\section{Abstract}

Recently the density peaks clustering algorithm (dubbed as DPC) attracts lots of attention. The DPC is able to quickly find cluster centers and complete clustering tasks. And the DPC is suitable for many clustering tasks. However, the cutoff distance $d d c c$ is depends on human experience which will greatly affect the clustering results. In addition, the selection of cluster centers requires manual participation which will affect the clustering efficiency. In order to solve these problem, we propose a density peaks clustering algorithm based on $\mathrm{K}$ nearest neighbors with adaptive merging strategy (dubbed as KNN$A D P C)$. We propose a clusters merging strategy to automatically aggregate the over-segmented clusters. Additionally, the $\mathrm{K}$ nearest neighbors is adopted to divide points more reasonably. The KNN-ADPC only has one parameter and the clustering task can be conducted automatically without human involvement. The experiment results on artificial and real-world datasets prove the higher accuracy of KNN-ADPC compared with DBSCAN, K-means++, DPC and DPC-KNN.

\section{Full Text}

This preprint is available for download as a PDF.

\section{Figures}

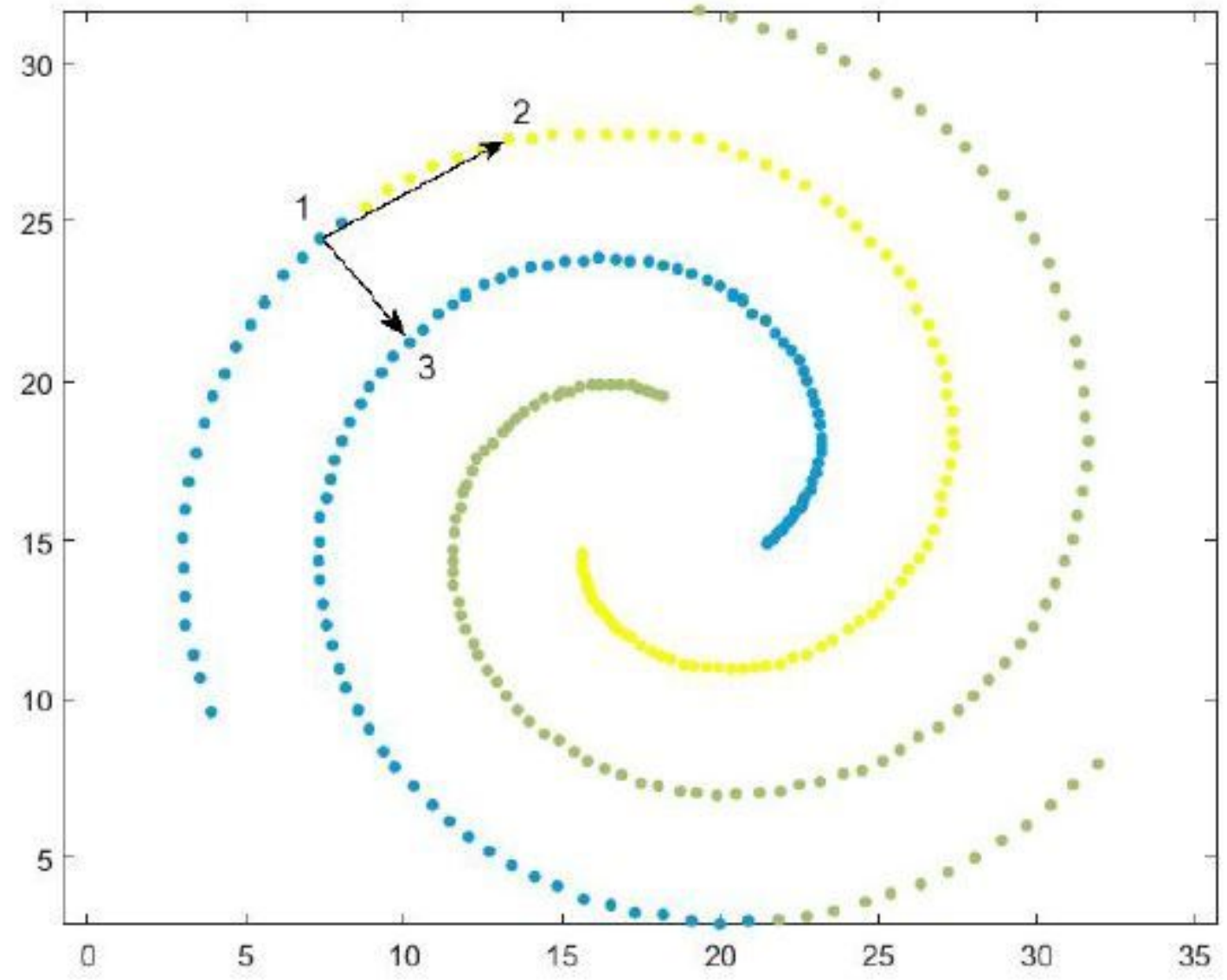


Figure 1

Misallocation diagram.

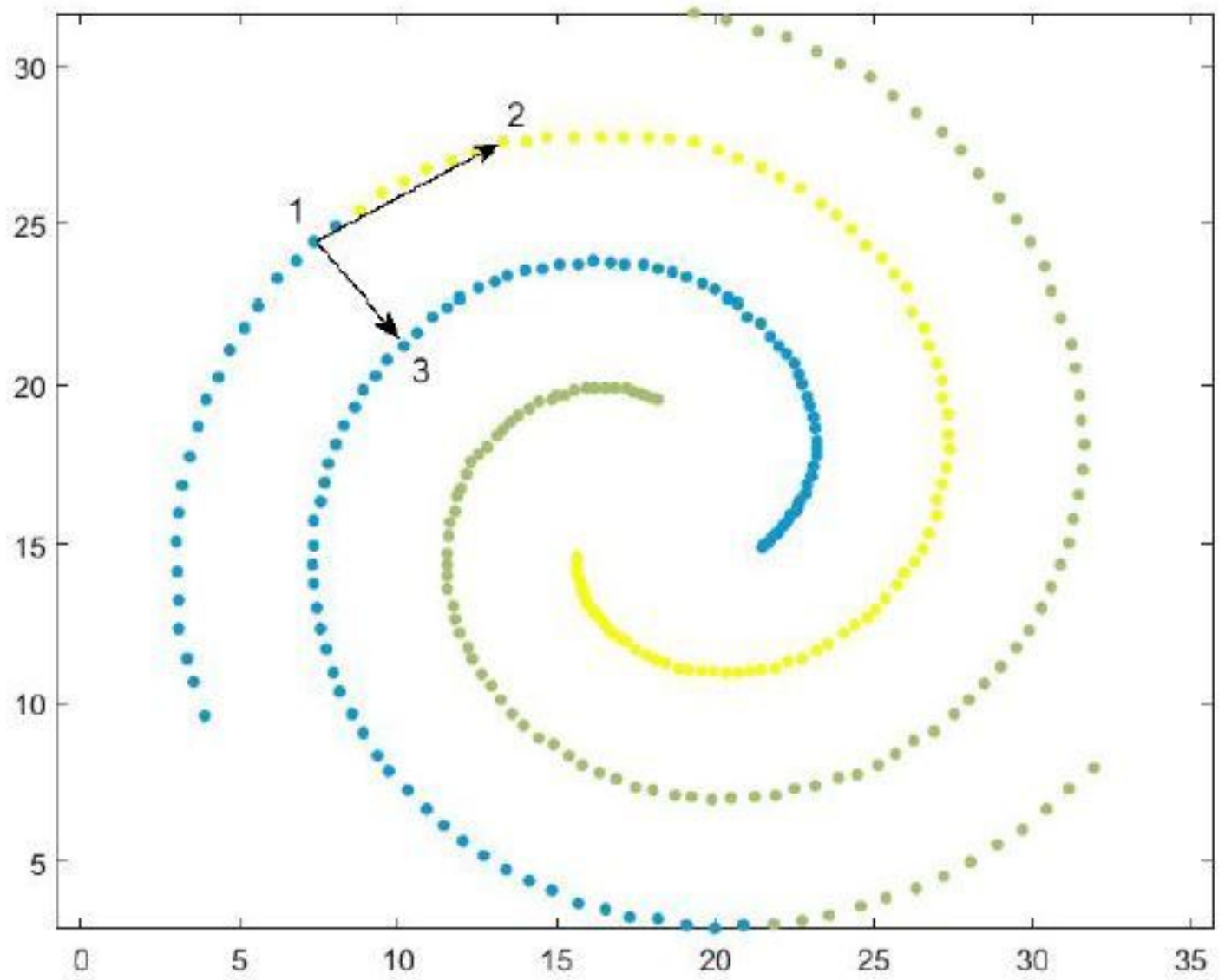

Figure 1

Misallocation diagram. 


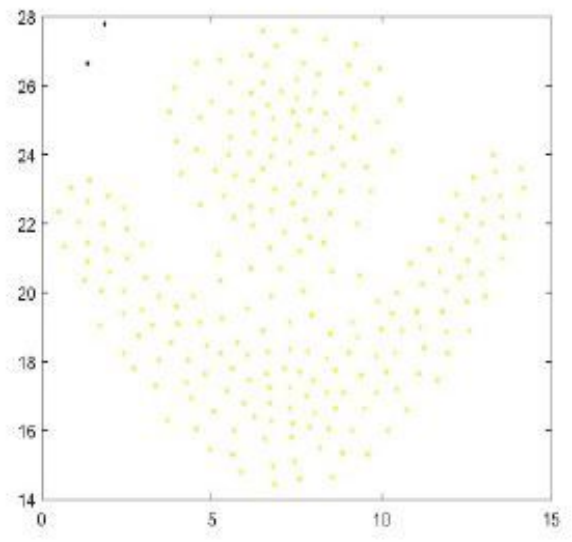

DBSCAN

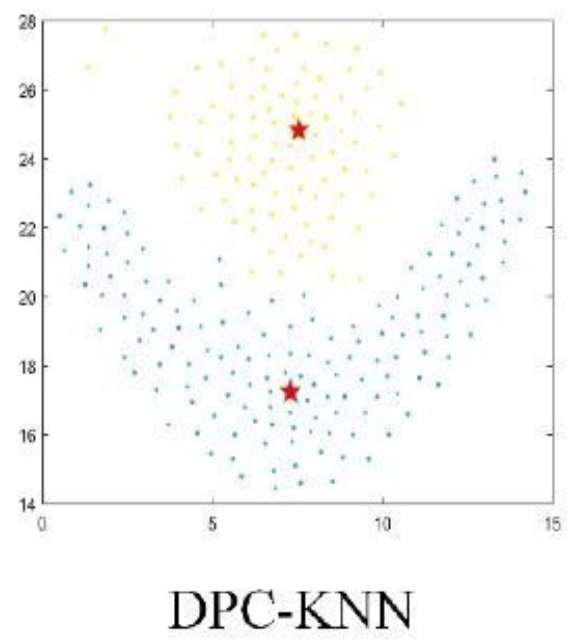

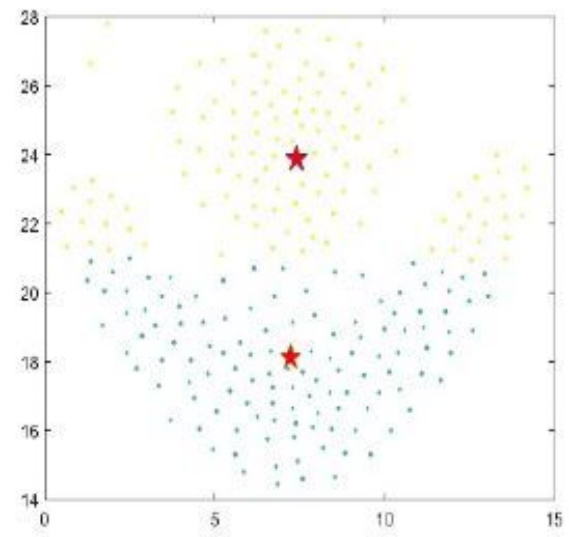

k-means++

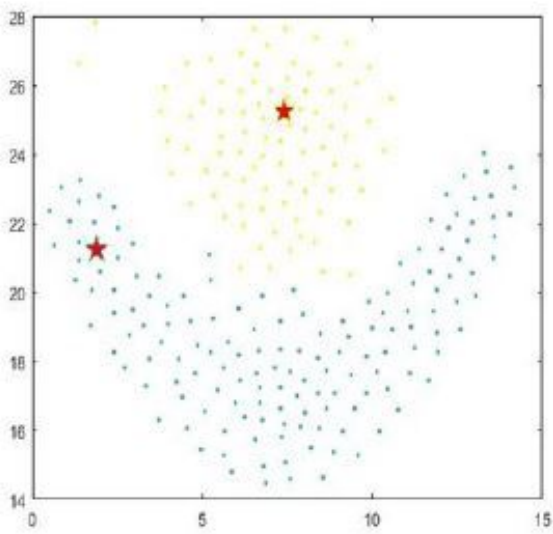

KNN-ADPC

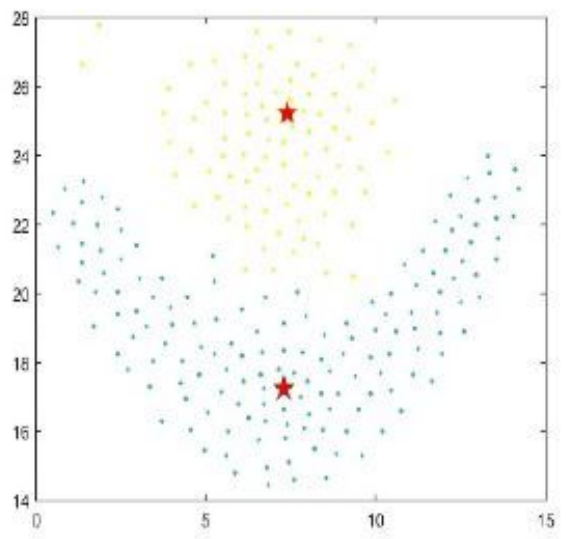

DPC

Figure 2

The performance of each algorithm on Flame. 


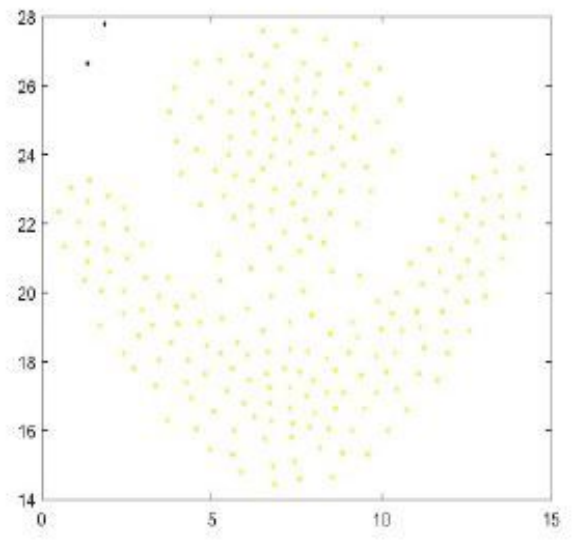

DBSCAN

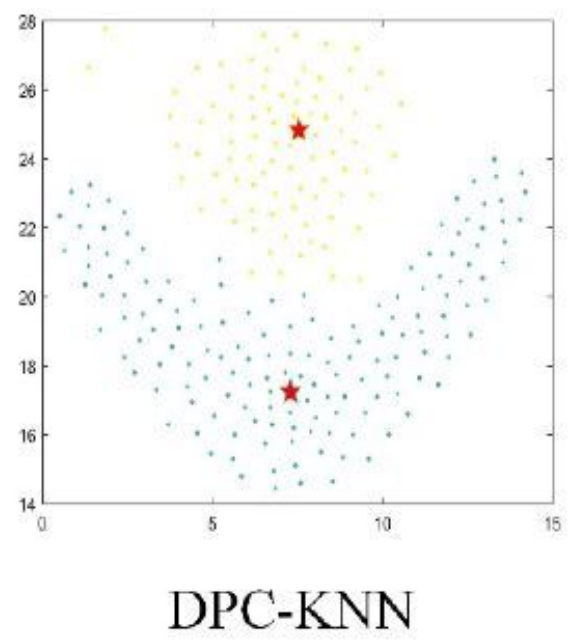

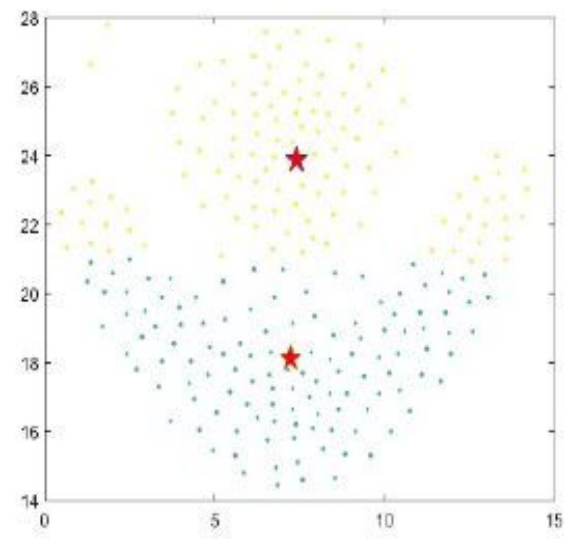

k-means++

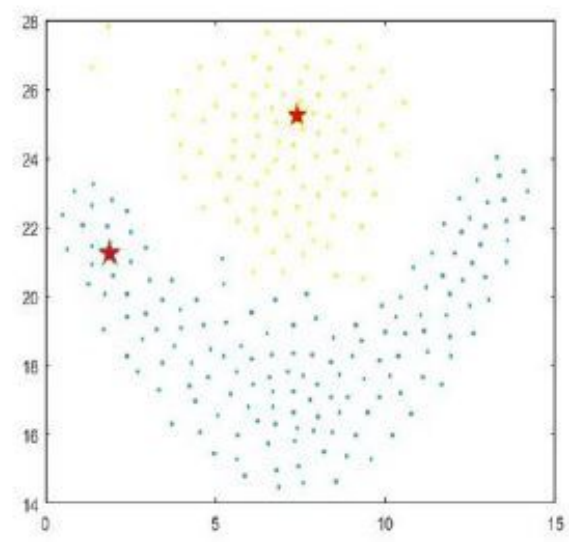

KNN-ADPC

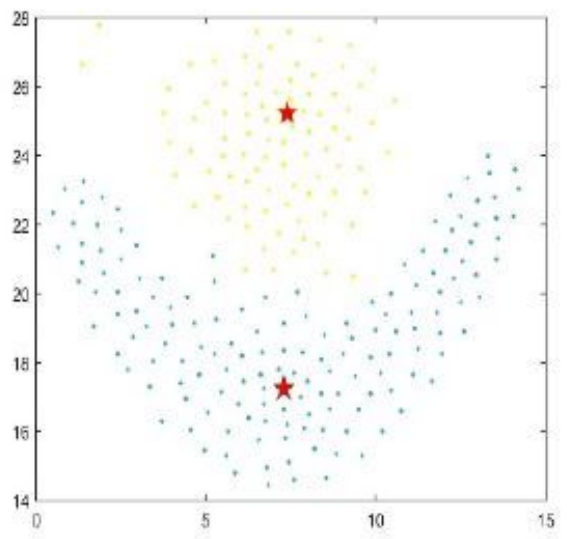

DPC

Figure 2

The performance of each algorithm on Flame. 


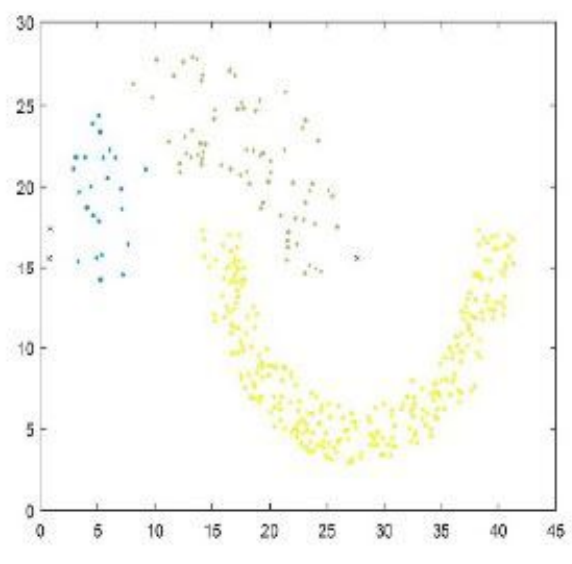

DBSCAN

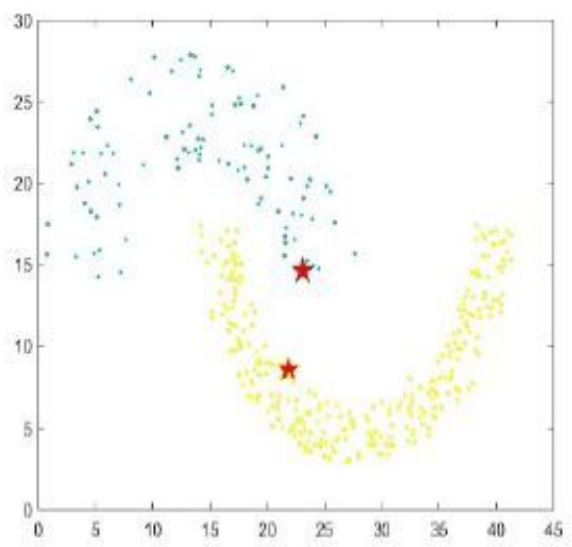

DPC-KNN

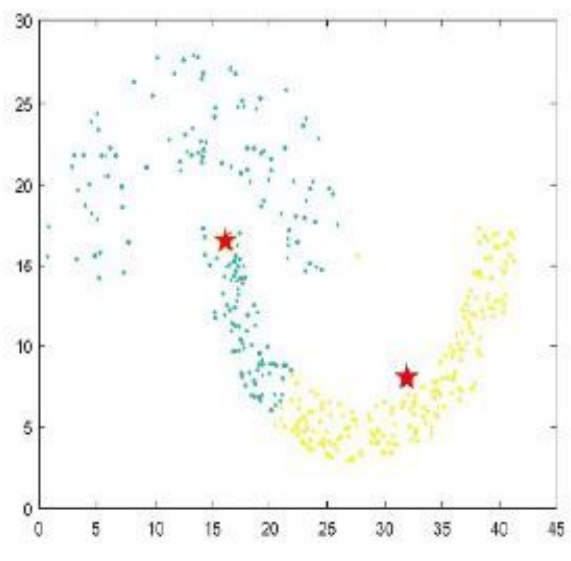

k-means++

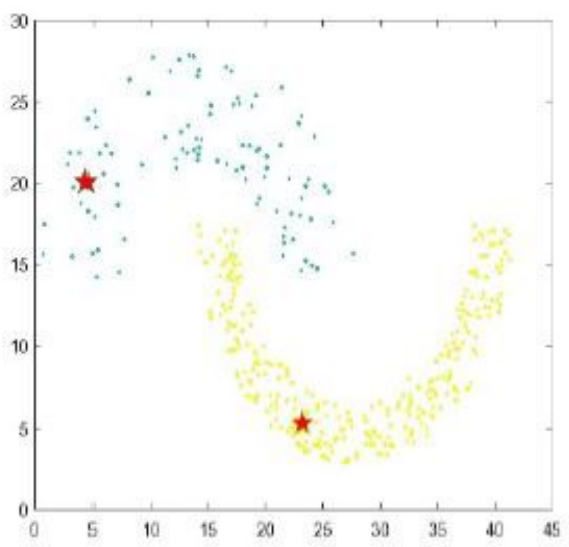

KNN-ADPC

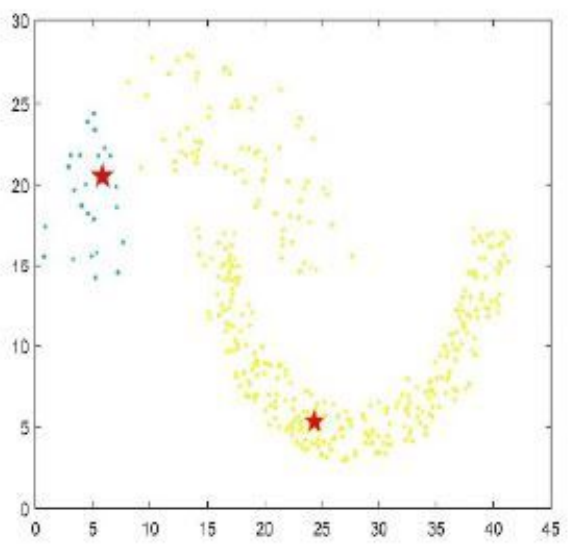

DPC

\section{Figure 3}

The performance of each algorithm on Jain. 


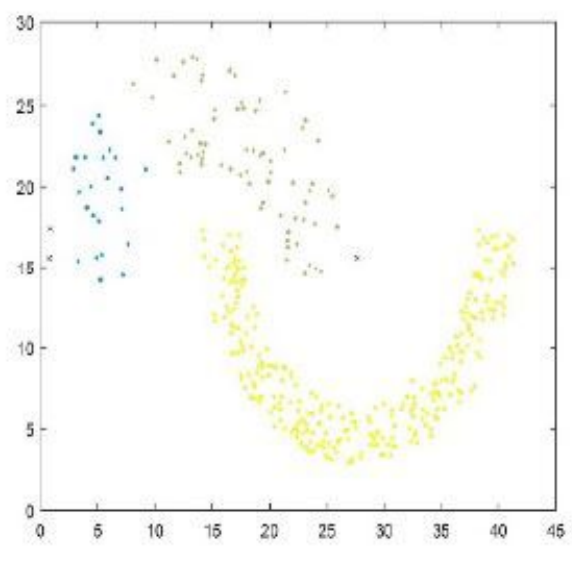

DBSCAN

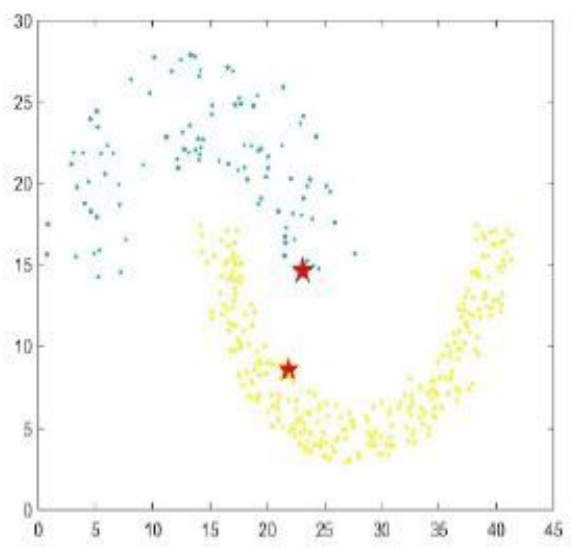

DPC-KNN

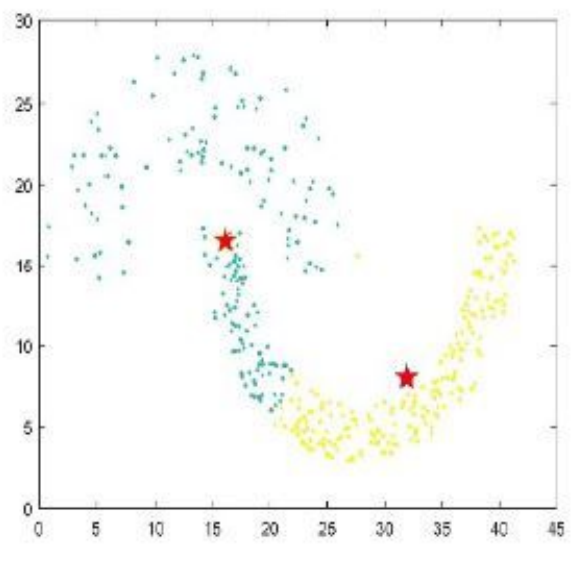

k-means++

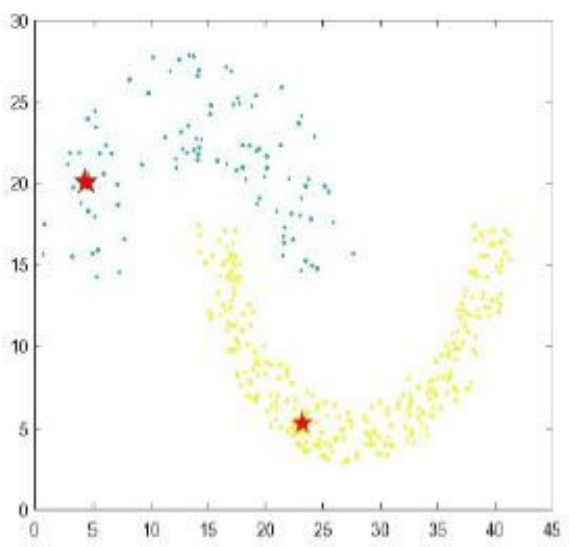

KNN-ADPC

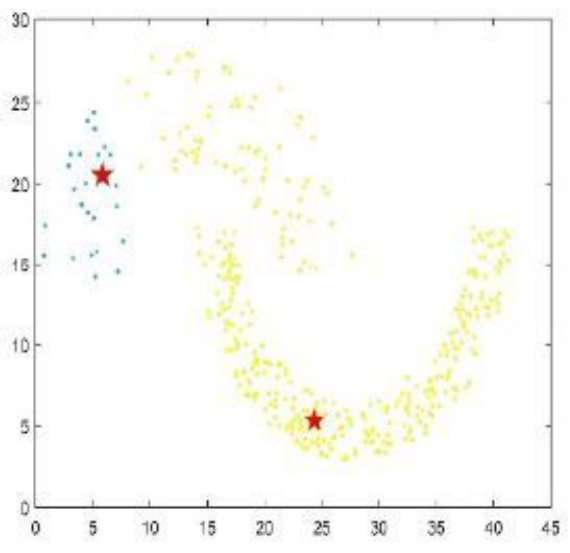

DPC

\section{Figure 3}

The performance of each algorithm on Jain. 


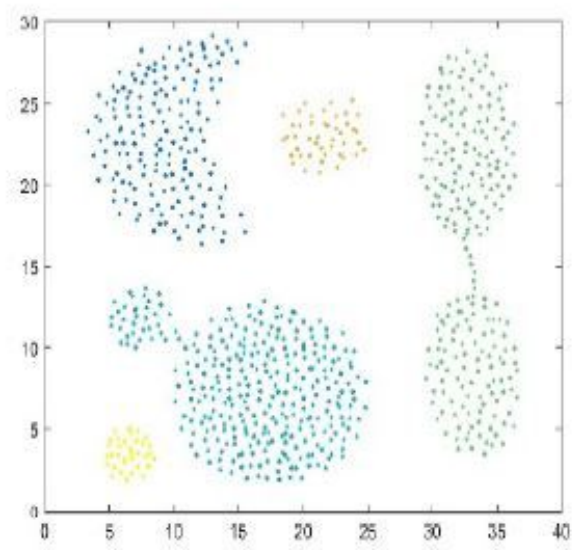

DBSCAN

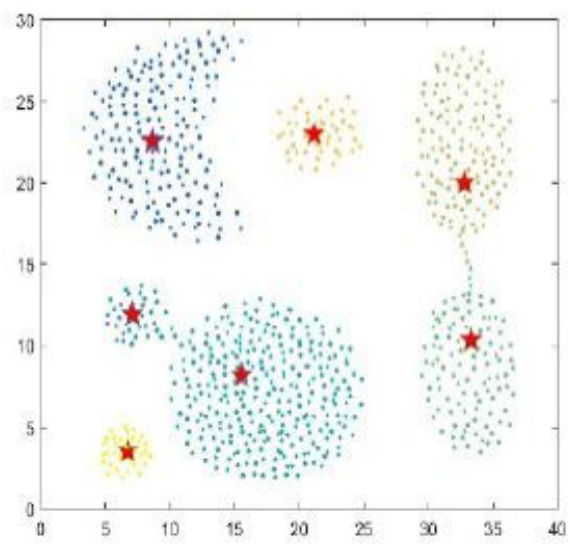

DPC-KNN

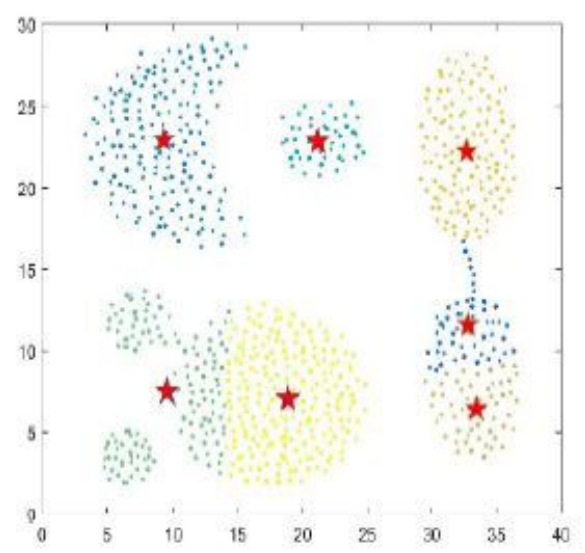

k-means ++

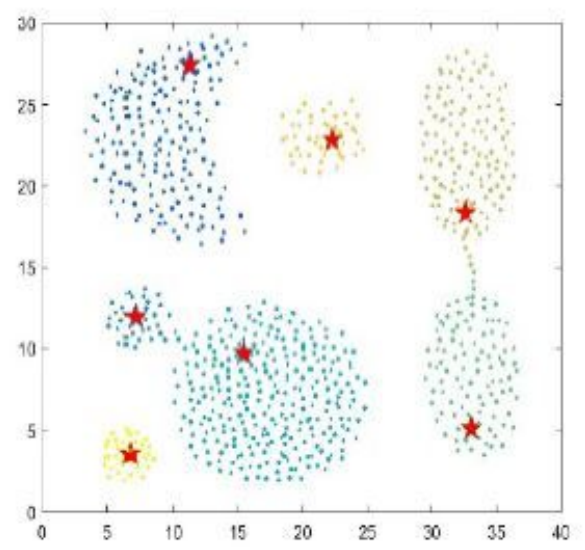

KNN-ADPC

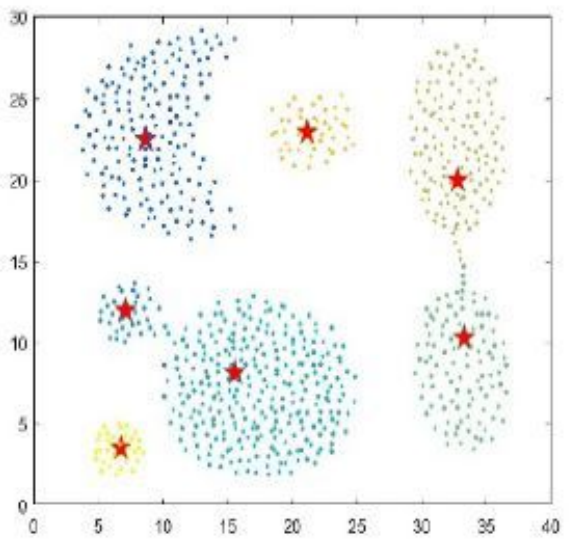

DPC

Figure 4

The performance of each algorithm on Aggregation. 


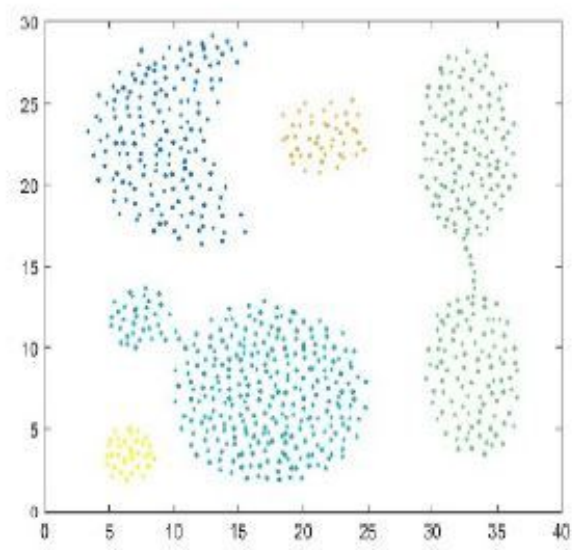

DBSCAN

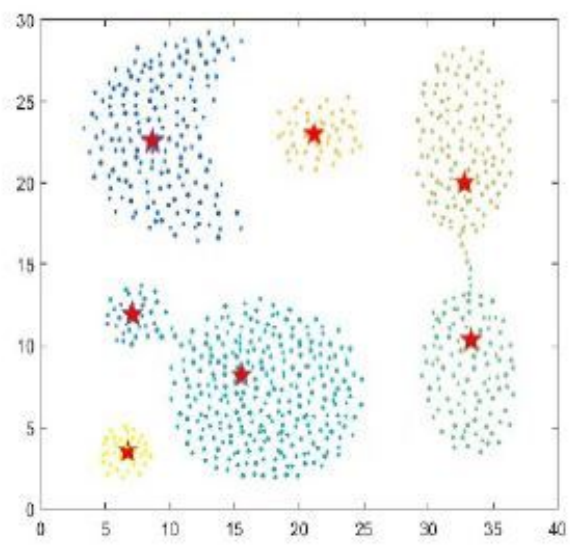

DPC-KNN

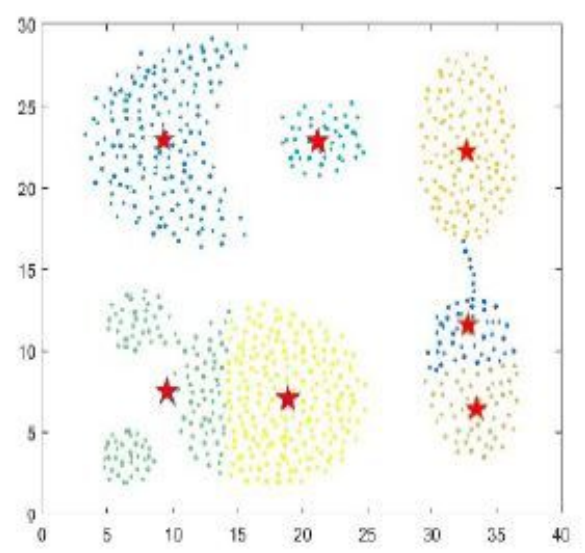

k-means ++

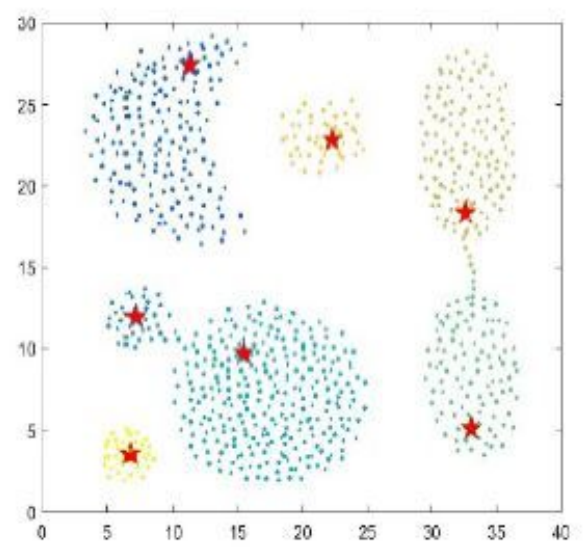

KNN-ADPC

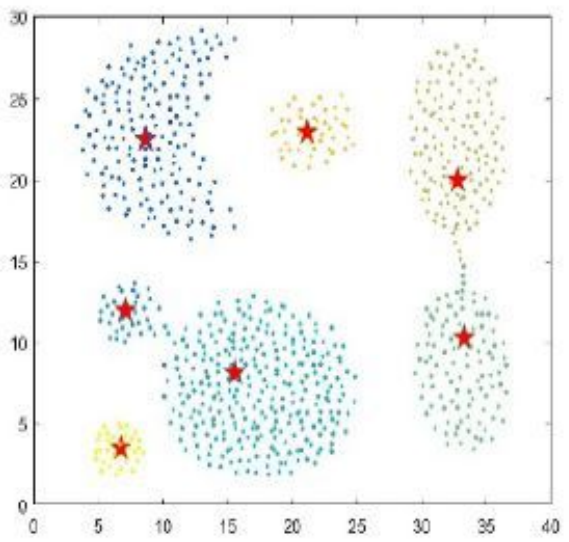

DPC

Figure 4

The performance of each algorithm on Aggregation. 


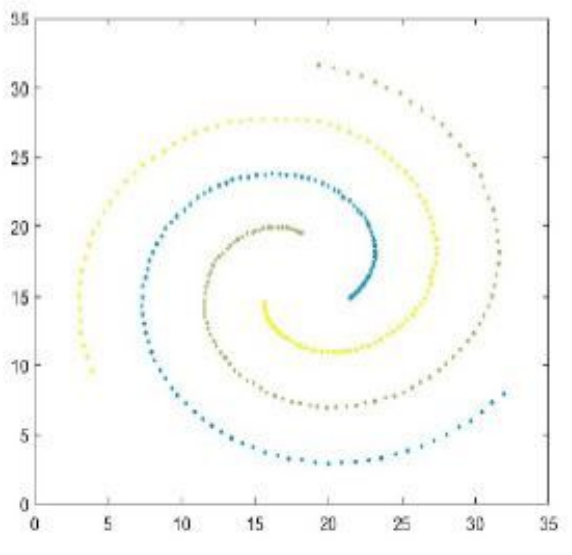

DBSCAN

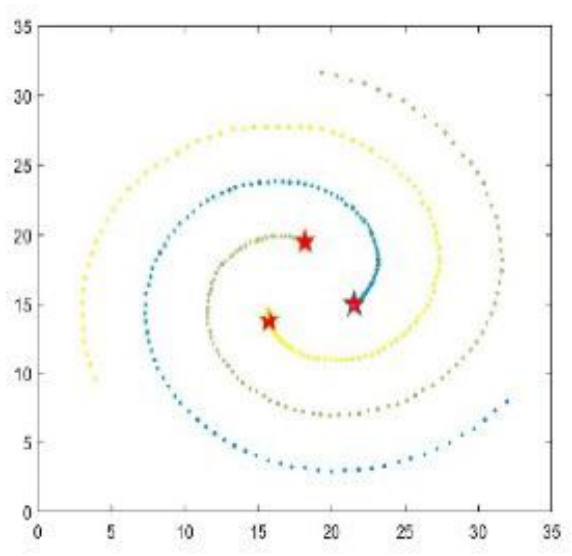

DPC-KNN

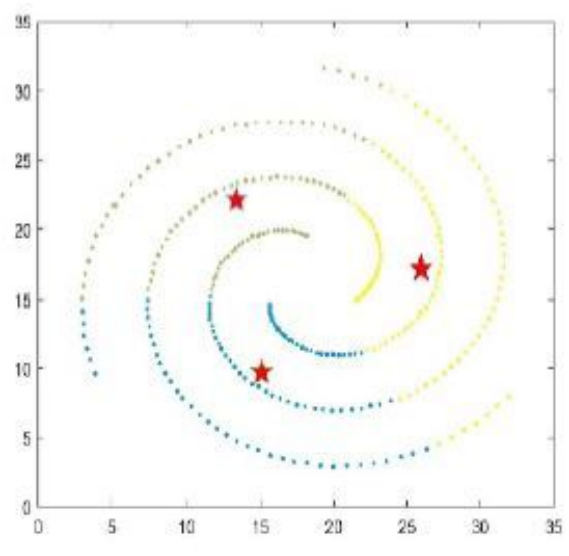

k-means++

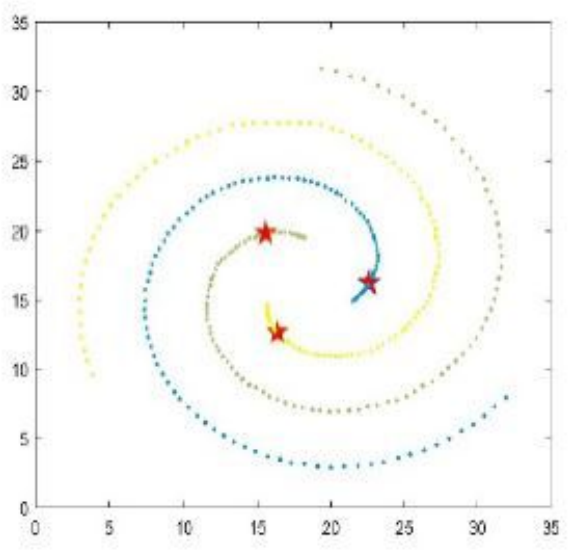

KNN-ADPC

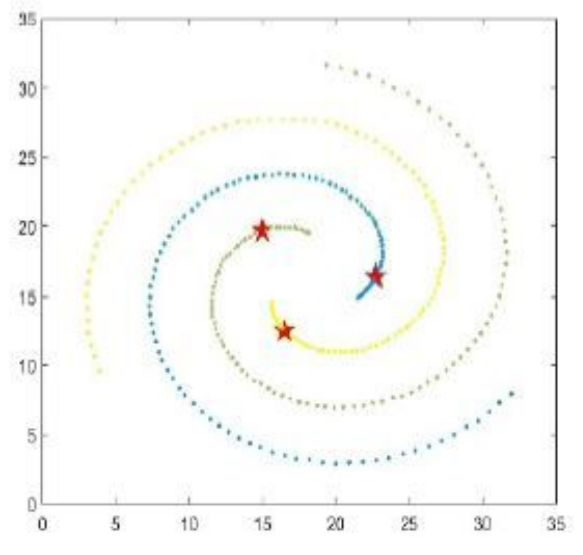

DPC

\section{Figure 5}

The performance of each algorithm on Spiral. 


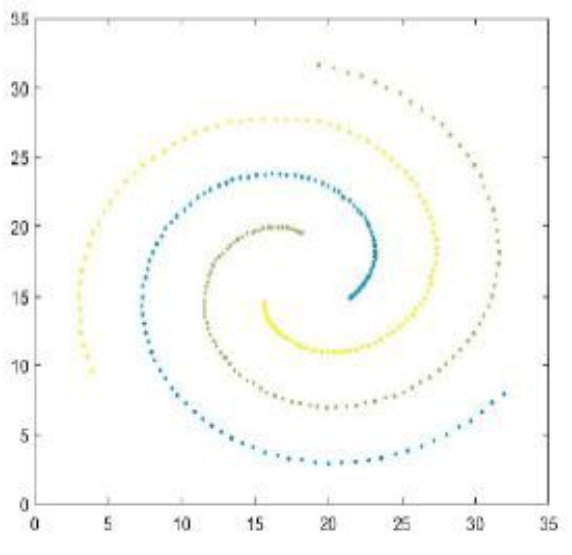

DBSCAN

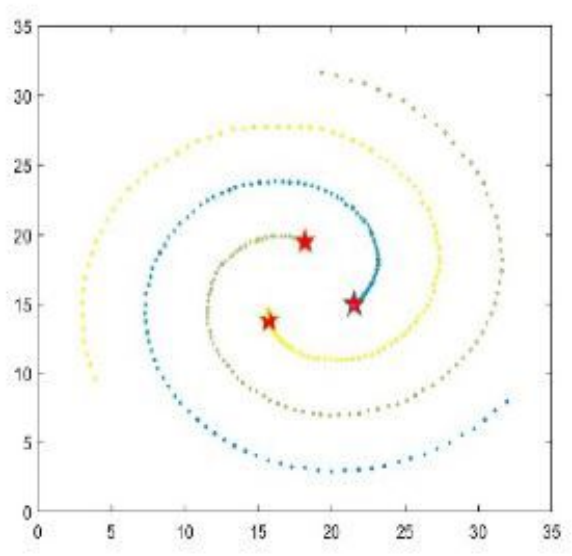

DPC-KNN

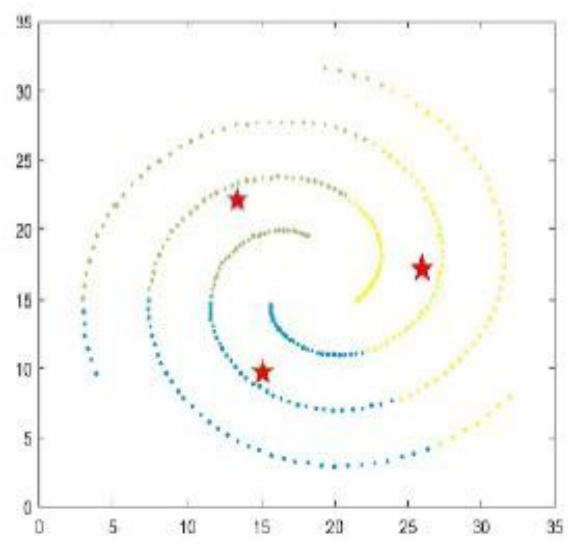

k-means++

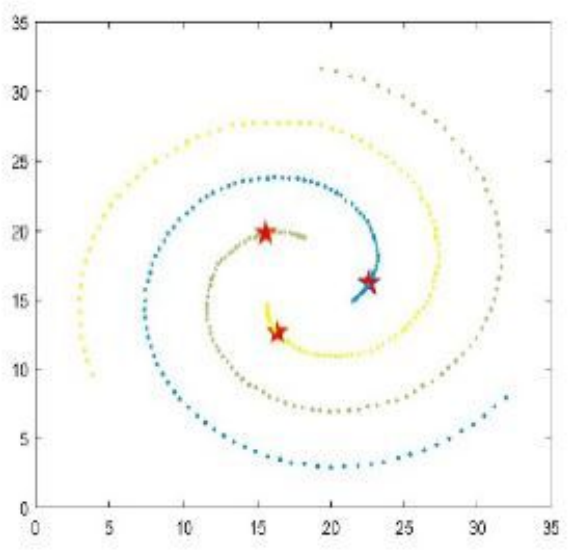

KNN-ADPC

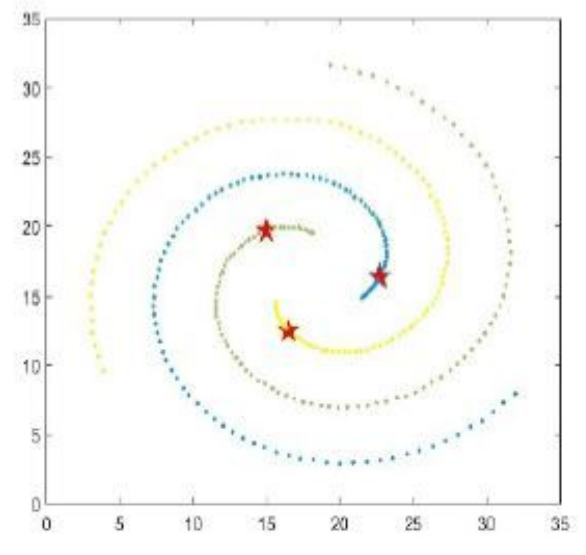

DPC

\section{Figure 5}

The performance of each algorithm on Spiral. 


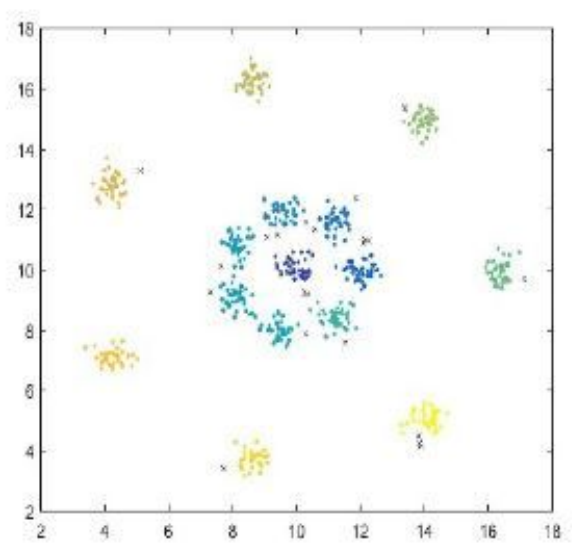

DBSCAN

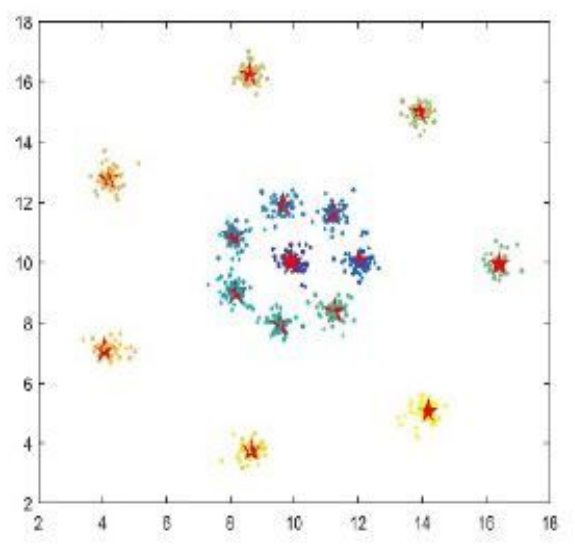

DPC-KNN

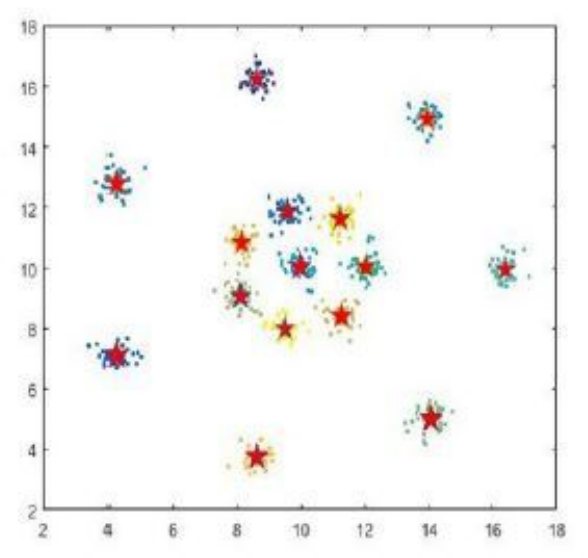

k-means ++

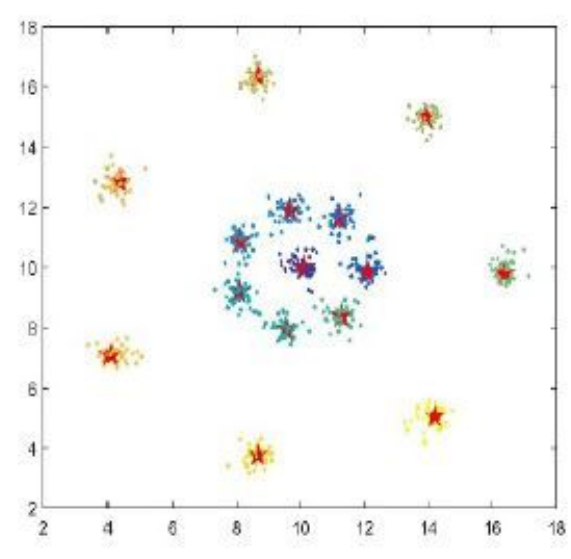

$\mathrm{KNN}-\mathrm{ADPC}$

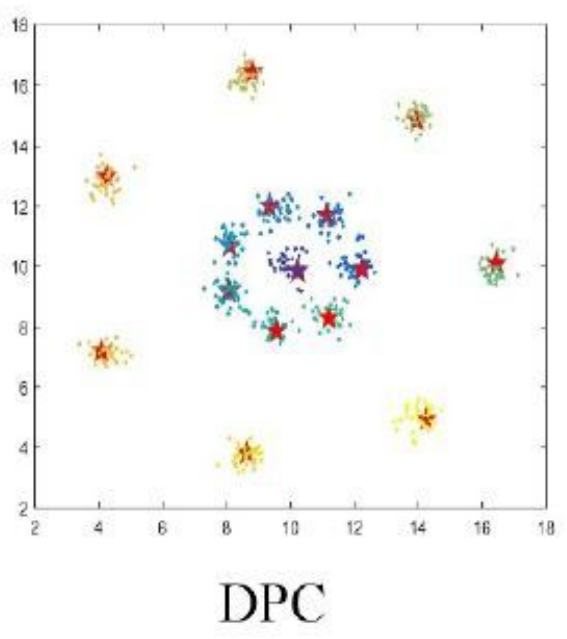

DPC

Figure 6

The performance of each algorithm on R15. 


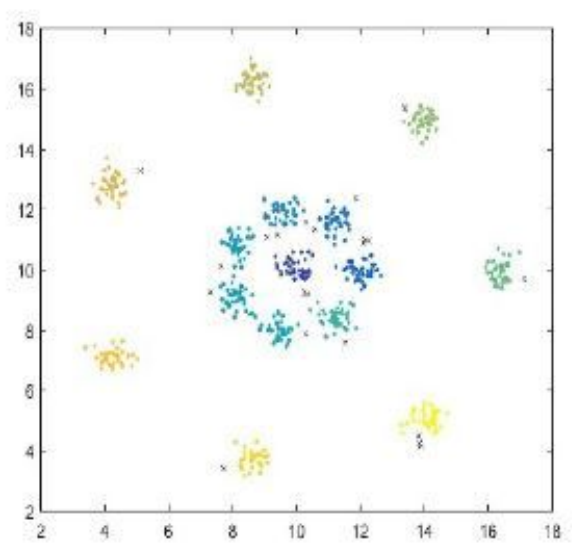

DBSCAN

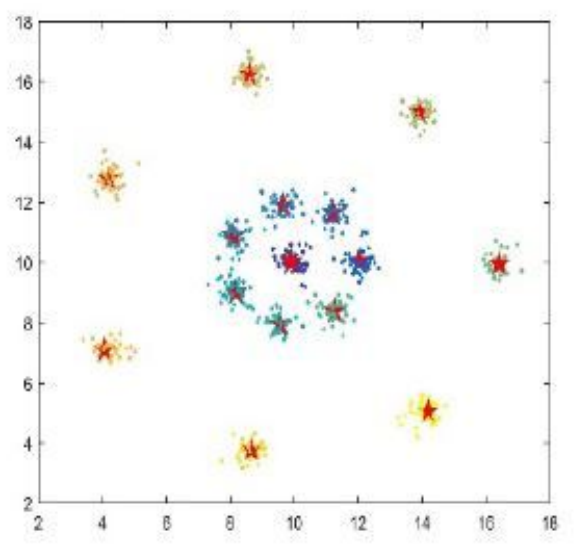

DPC-KNN

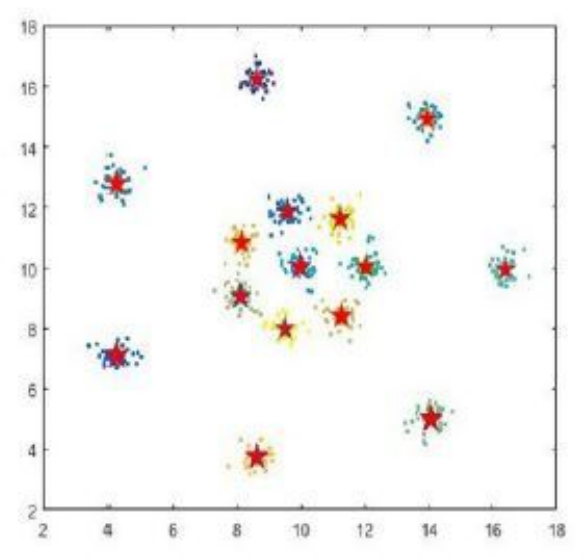

k-means ++

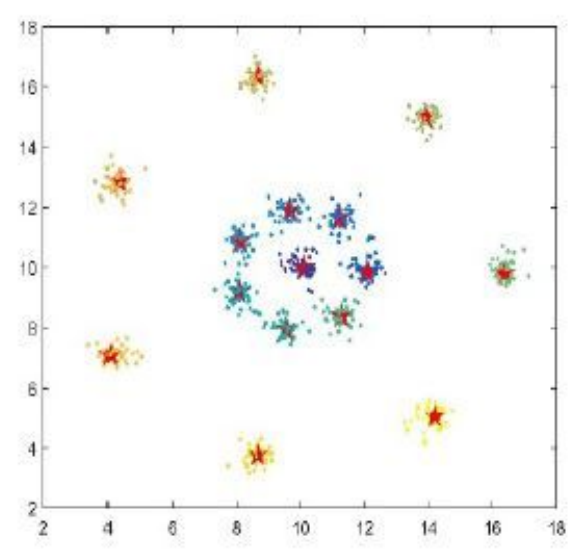

$\mathrm{KNN}-\mathrm{ADPC}$

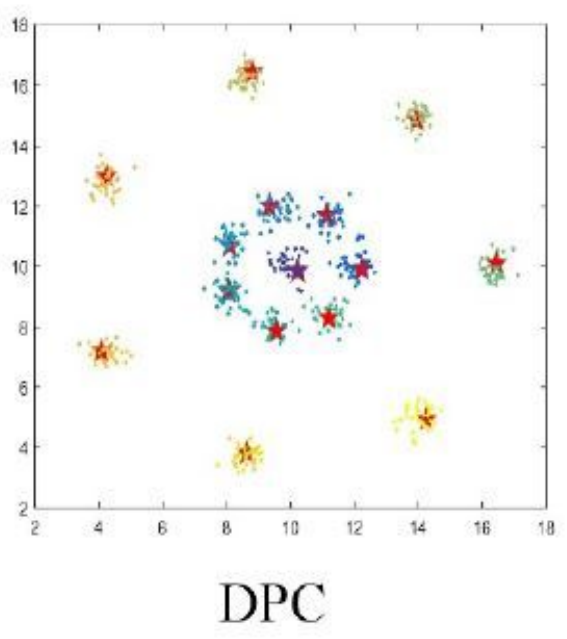

DPC

Figure 6

The performance of each algorithm on R15. 


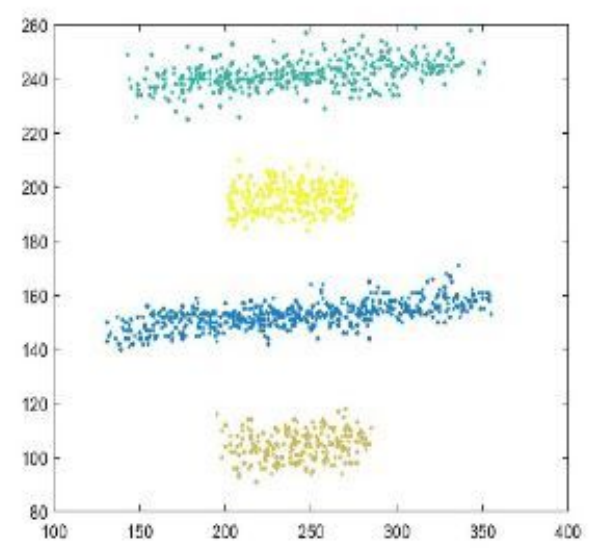

DBSCAN

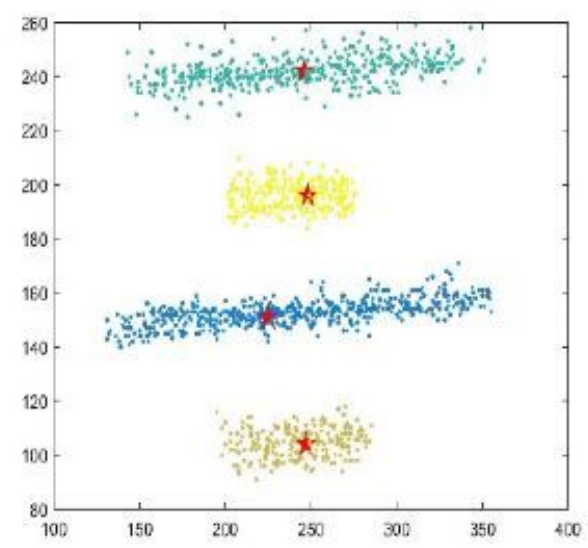

DPC-KNN

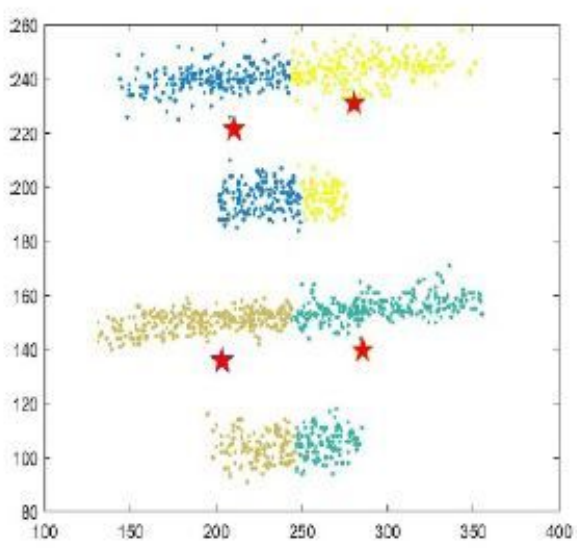

k-means++

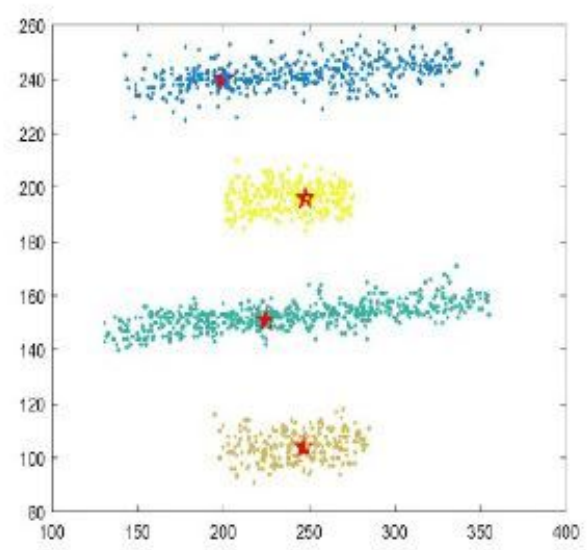

KNN-ADPC

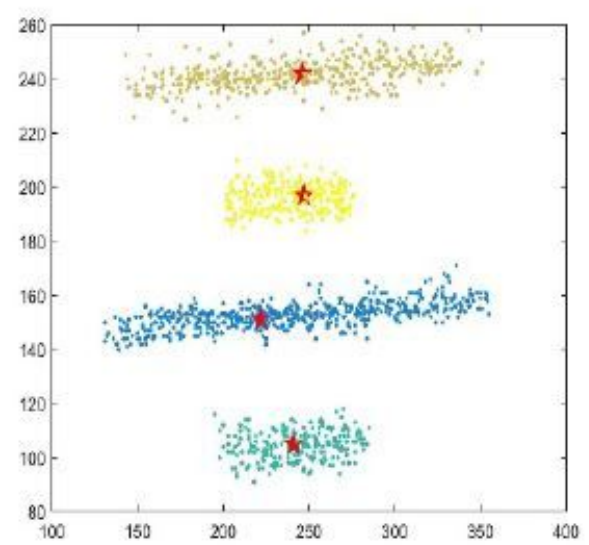

DPC

\section{Figure 7}

The performance of each algorithm on D4. 


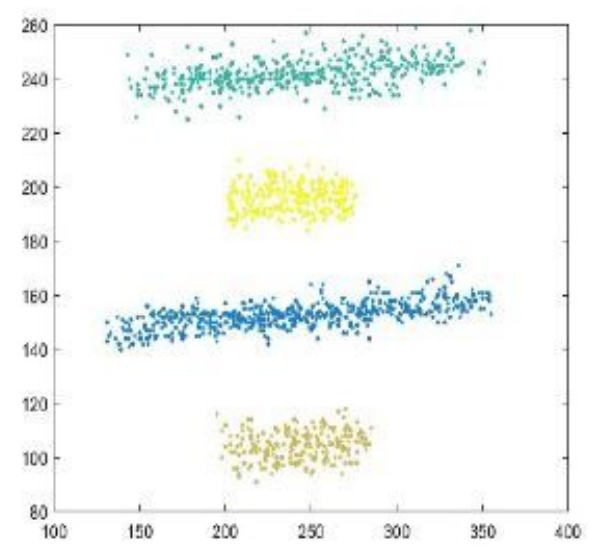

DBSCAN

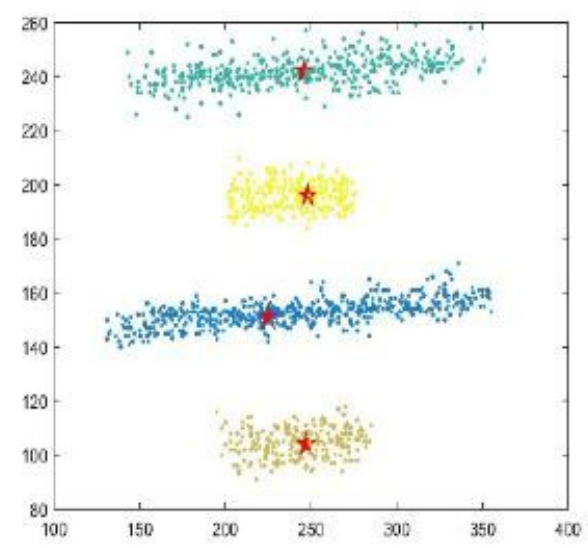

DPC-KNN

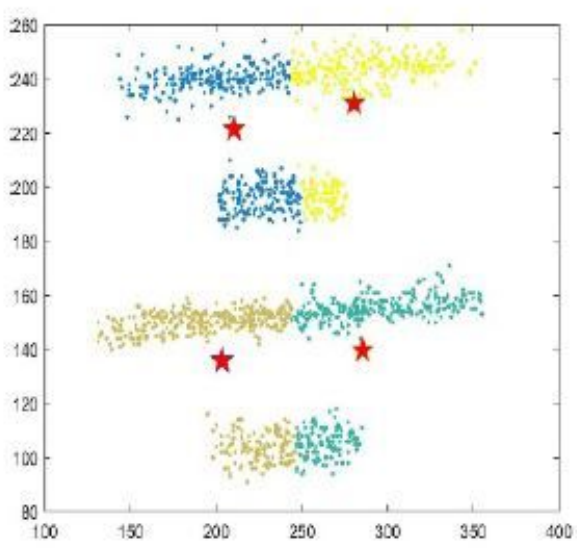

k-means++

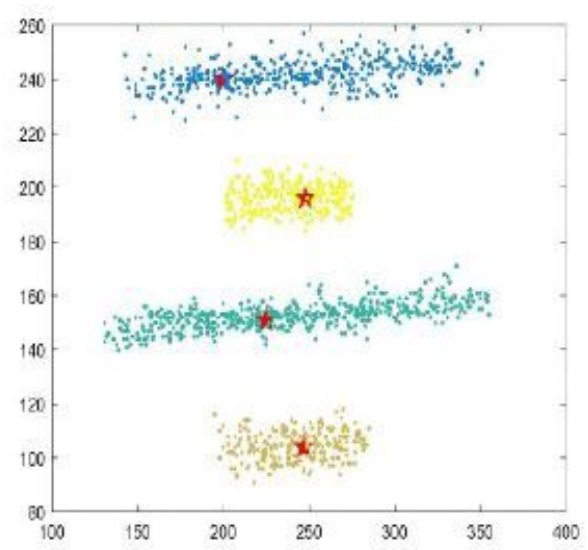

KNN-ADPC

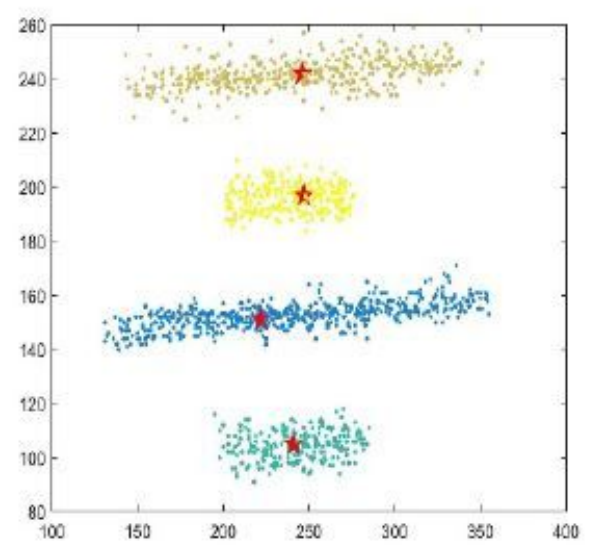

DPC

\section{Figure 7}

The performance of each algorithm on D4. 


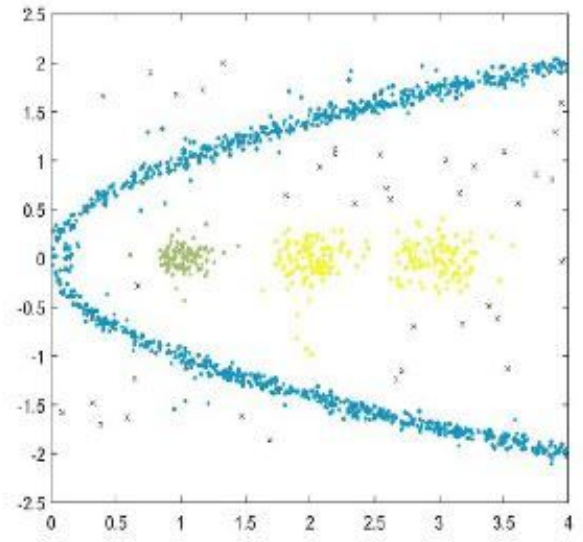

DBSCAN

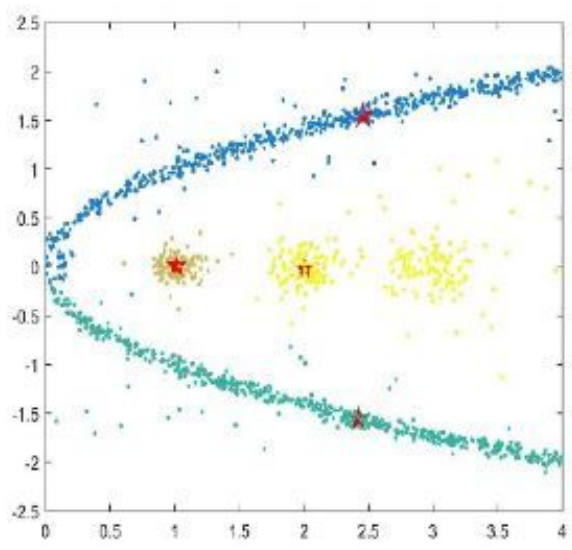

DPC-KNN

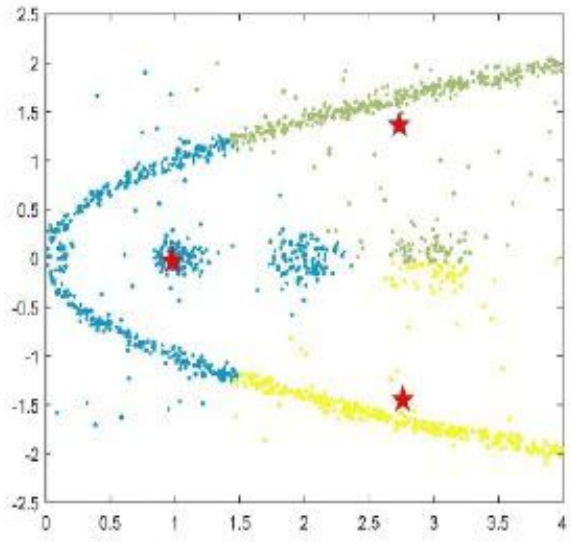

k-means++

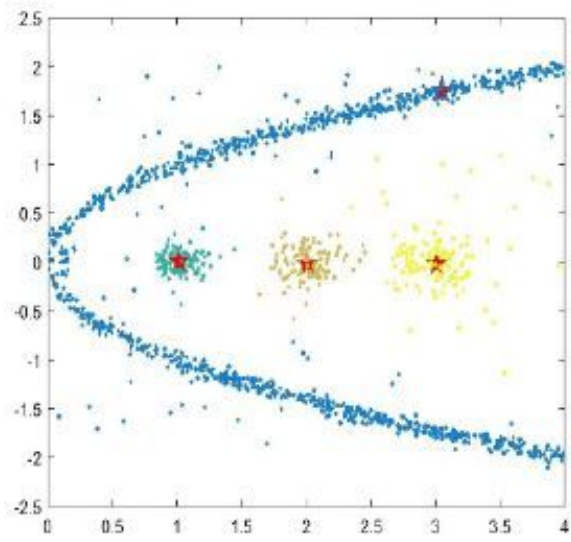

KNN-ADPC

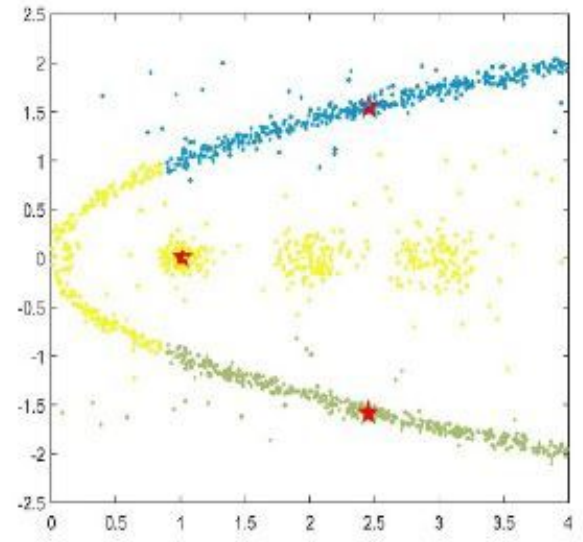

DPC

\section{Figure 8}

The performance of each algorithm on D9. 


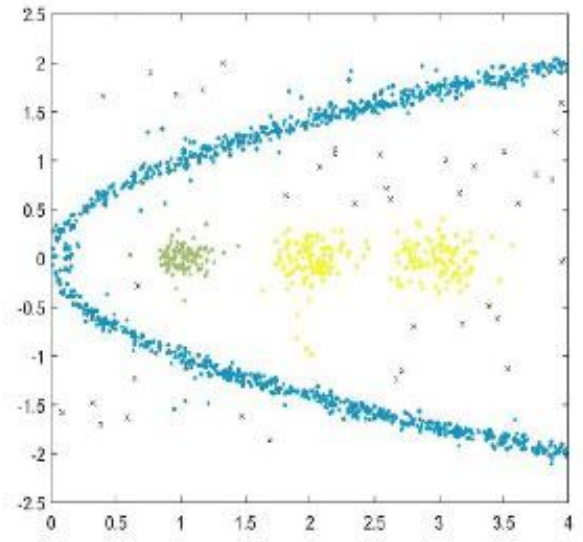

DBSCAN

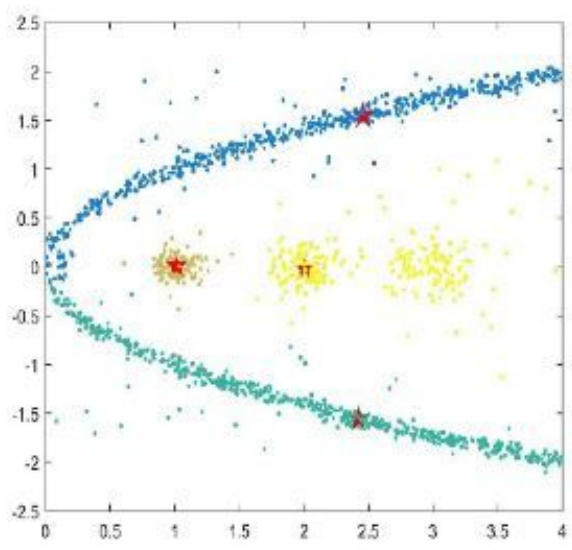

DPC-KNN

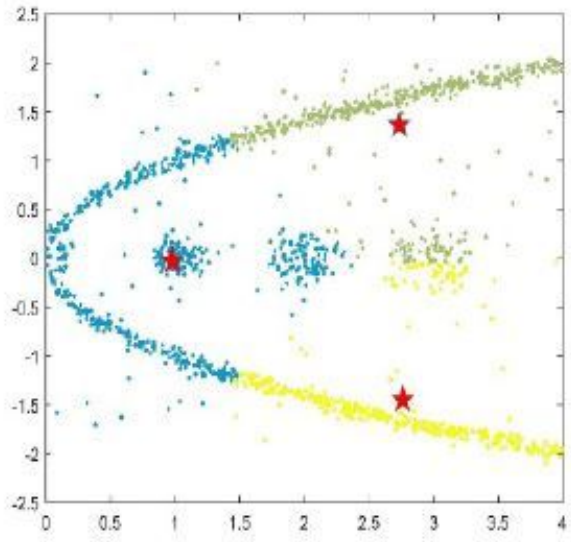

k-means++

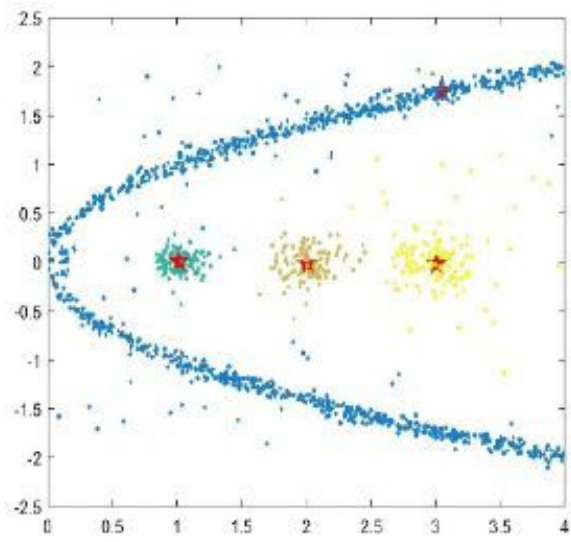

KNN-ADPC

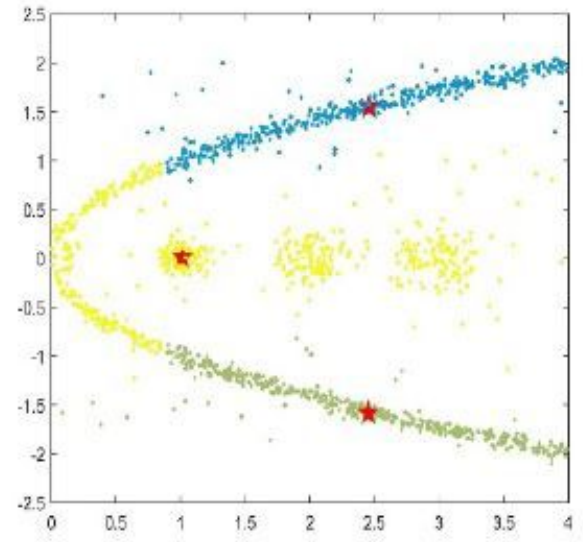

DPC

\section{Figure 8}

The performance of each algorithm on D9. 